\title{
Papers
}

\section{Child psychiatric disorder and relative age within school year: cross sectional survey of large population sample}

\author{
Robert Goodman, Julia Gledhill, Tamsin Ford
}

\begin{abstract}
Objective To test the hypothesis that younger children in a school year are at greater risk of emotional and behavioural problems.

Design Cross sectional survey.

Setting Community sample from England, Scotland, and Wales.

Participants 10438 British 5-15 year olds.

Main outcome measures Total symptom scores on psychopathology questionnaires completed by parents, teachers, and 11-15 year olds; psychiatric diagnoses based on a clinical review of detailed interview data.

Results Younger children in a school year were significantly more likely to have higher symptom scores and psychiatric disorder. The adjusted regression coefficients for relative age were $0.51(95 \%$ confidence interval 0.36 to $0.65, \mathrm{P}<0.0001)$ according to teacher report and $0.35(0.23$ to $0.47, \mathrm{P}=0.0001)$ for parental report. The adjusted odds ratio for psychiatric diagnoses for decreasing relative age was 1.14 (1.03 to $1.25, \mathrm{P}=0.009$ ). The effect was evident across different measures, raters, and age bands. Cross national comparisons supported a "relative age" explanation based on the disadvantages of immaturity rather than a "season of birth" explanation based on seasonal variation in biological risk.

Conclusions The younger children in a school year are at slightly greater psychiatric risk than older children. Increased awareness by teachers of the relative age of their pupils and a more flexible approach to children's progression through school might reduce the number of children with impairing psychiatric disorders in the general population.
\end{abstract}

\section{Introduction}

Many studies have shown that the youngest children in a school year tend to be disadvantaged by the educational system..$^{1-3}$ This is confirmed by our own analyses of data from a large community sample, in which we found that the youngest children in the school year were at an increased risk of being incorrectly identified by teachers as having learning difficulties. ${ }^{4}$ As different countries use different cut-off dates for school entry, national comparisons are illuminating. Whereas children born between September and December are at an advantage in England, where they are the oldest in their class, children born in these months are at a disadvantage in Sweden, where they are the youngest in their class. ${ }^{5}$ This is strong evidence for a "relative age" explanation based on the disadvantage of youth rather than a "season of birth" explanation based on seasonal variation in biological risk-for example, for prenatal infection. The educational disadvantage experienced by the youngest children in a class is not confined to the early school years but persists into secondary education and influences university entrance. ${ }^{26}$

A study conducted in London more than 20 years ago suggested that "relative age" also influenced the rate of mental health problems in children. ${ }^{7}$ We have re-examined this association by using recent data from a large epidemiological survey of children's mental health in Britain. ${ }^{8}$ As "season of birth" has previously been linked to mental health problems, ${ }^{9}$ we used the different cut-off dates for school entry in Scotland and in England and Wales as a "natural experiment" to evaluate the likely cause of psychological disadvantage for the youngest children.

\section{Methods}

We used data collected in 1999 on a nationally representative sample of British 5-15 year olds. ${ }^{8}$ The child benefit register was used to develop a sampling frame of postal sectors within England, Wales, and Scotland. Four hundred and seventy five postal sectors, stratified by regional health authority and socioeconomic group, were sampled with a probability related to the size of the sector; the response rate was $83 \%$.

In England and Wales, the cut-off date for school entry is 1 September; children must start school in the academic year during which they will become 5 years old. We followed the educational tradition of dividing children into autumn-born (the oldest third in the class, with birthdays in September to December), spring-born (with birthdays in January to April), and summer-born (the youngest third in the class, with birthdays in May to August) groups. In Scotland the cut-off date for determining school entry is 1 March, and the academic year begins in August. Children with birthdays in March to August must start school in the academic year during which they will become 5, whereas children with birthdays in September to February can start in the August preceding their fifth birthday or defer for a year. The proportion who defer
Department of Child and Adolescent Psychiatry, Institute of Psychiatry, King's College London, College London,
London SE5 8AF Robert Goodman professor

Tamsin Ford clinical research fellow Child and Adolescent Psychiatry, Imperial College School of Medicine (St Mary's), London Mary's), I
W2 1PG Julia Gledhill clinical research fellow Correspondence to: R Goodman r.goodman@ iop.kcl.ac.uk

bmj.com 2003;327:472 
is not centrally recorded, but inquiries to several local education authorities in Scotland indicated that the proportion of children deferring was around 1-12\% in 2000-1 and was probably lower still in 1999 when the survey was conducted. We classified the Scottish sample into the oldest third (birthdays in March to June), the middle third (July to October), and the youngest third (November to February). As data on which children deferred school entry were not collected in our sample, we will have misclassified the relative age of a small number of children who deferred. For this reason, and also because the number of children in the Scottish sample was much smaller than that for England and Wales, we give greater emphasis in the analysis to the data for England and Wales. It is rare for children to repeat an academic year in the United Kingdom.

Our outcome measures included a dimensional measure of symptoms according to parents, teachers, and self report and the presence or absence of at least one psychiatric diagnosis. ${ }^{10}$ The strengths and difficulties questionnaire is a well validated questionnaire that asks about children's emotions, behaviour, activity levels, peer relationships, and pro-social behaviour; it generates a total symptoms score. ${ }^{11}$ Parents, teachers, and children aged 11 and over completed the strengths and difficulties questionnaire, but younger children were not asked to complete a questionnaire as they do not generally provide reliable reports. ${ }^{8}$

The development and wellbeing assessment consists of a structured interview administered by lay interviewers, who also recorded verbatim accounts of any reported problems. ${ }^{12}$ All parents and children aged 11 or over were interviewed, and a shortened version of the development and wellbeing assessment was posted to the child's teacher. Experienced clinicians used the transcripts combined with the symptom and impairment scores from all the available informants to make diagnoses according to internationally recognised criteria, ${ }^{10}$ emulating the clinical process as closely as possible. A disorder was diagnosed only if the symptoms had an important impact, in terms of distress or interference with the child's everyday life. Use of strict impact criteria led to a conservative prevalence rate of $9.5 \%$ for all psychiatric disorders, ${ }^{8}$ which is towards the lower end of the range established by previous studies. ${ }^{13}$

We examined the relation between relative age and psychopathology by using $\chi^{2}$ for trend and logistic regression for categorical outcomes (psychiatric disorder) and correlations and linear regression for dimensional outcomes (total difficulties scores). We included variables in the multivariate analysis if previous studies or prior analysis had shown them to be associated with psychiatric disorder or higher levels of psychopathology.

\section{Results}

Data were collected from 10438 British children aged 5-15 years. Of these, 9383 (89.9\%) children were living in England and Wales. The mean age of the total sample was 9.9 years. Being in the youngest third of the class was not significantly associated with any other sociodemographic (sex, ethnic group, social class) or family (number of children in the household, maternal educational level, family type) characteristics.

The results shown in the table for England and Wales show a significant increase in risk of psychopathology with decreasing relative age. This finding was not confined to younger children but persisted into those of secondary school age.

Multivariate analyses showed that relative age remained an independent risk factor for psychiatric disorder (adjusted odds ratio for relative age 1.14, 95\% confidence interval 1.03 to 1.25) after adjustment for age, sex, maternal educational level, family type, number of children at home, maternal psychopathology, family dysfunction, IQ of child, and specific learning difficulties. The adjusted regression coefficients for relative age according to teacher reported difficulties (0.51, 0.36 to $0.65, \mathrm{P}=0.0001)$, parent reported difficulties $(0.35,0.23$ to $0.47, \mathrm{P}=0.0001)$, and self reported difficulties $(0.23,0.03$ to $0.43, \mathrm{P}=0.03)$ showed a similar relation with symptom scores regardless of informant.

The figure shows that the Scottish data look very similar to the data from England and Wales when plotted in terms of relative age. This was not so when we replotted the data in terms of season of birth (not

Percentage of children with any psychiatric disorder and mean symptom score on the parent, teacher, and self completed strengths and difficulties questionnaire by relative age for England and Wales

\begin{tabular}{|c|c|c|c|c|}
\hline Outcome measure & Oldest third $(n=3134)$ & Middle third $(\mathrm{n}=3014)$ & Youngest third $(\mathrm{n}=3235)$ & Significance \\
\hline \multicolumn{5}{|l|}{ All ages } \\
\hline Any psychiatric diagnosis* $(\mathrm{n}=9383)$ & 8.3 (7.4 to 9.3$)$ & 8.8 (7.8 to 9.8$)$ & 9.9 (8.9 to 10.9$)$ & $\chi^{2}=4.58, P=0.03$ \\
\hline Parent reported symptoms† $(\mathrm{n}=9267)$ & $8.2(8.0$ to 8.4$)$ & $8.4(8.2$ to 8.6$)$ & $8.8(8.6$ to 9.0$)$ & $r=0.05, \mathrm{P}<0.001$ \\
\hline Teacher reported symptoms† $(n=7452)$ & $6.2(5.9$ to 6.4$)$ & $6.6(6.3$ to 6.8$)$ & $7.0(6.8$ to 7.3$)$ & $r=0.06, \mathrm{P}<0.001$ \\
\hline Self reported symptoms† $(n=3799)$ & $10.2(9.9$ to 10.4$)$ & 10.4 (10.1 to 10.7$)$ & $10.6(10.4$ to 10.9$)$ & $r=0.04, \mathrm{P}=0.02$ \\
\hline Ages 5-10 & $(n=1786)$ & $(n=1712)$ & $(n=1843)$ & \\
\hline Any psychiatric diagnosis* $(\mathrm{n}=5341)$ & $7.4(6.2$ to 8.4$)$ & 7.1 (5.9 to 8.3$)$ & 8.5 (7.2 to 9.8$)$ & $\chi^{2}=1.34, P=0.2$ \\
\hline Parent reported symptoms† $(\mathrm{n}=5288)$ & $8.3(8.0$ to 8.6$)$ & $8.5(8.2$ to 8.8$)$ & $9.0(8.8$ to 9.3$)$ & $r=0.05, \mathrm{P}<0.001$ \\
\hline Teacher reported symptoms $\uparrow(n=4371)$ & 6.2 (5.9 to 6.5$)$ & 6.7 (6.4 to 7.0$)$ & $7.3(7.0$ to 7.6$)$ & $r=0.08, P<0.001$ \\
\hline Ages 11-15 & $(n=1348)$ & $(n=1302)$ & $(n=1392)$ & \\
\hline Any psychiatric diagnosis* $(\mathrm{n}=4042)$ & $9.5(7.9$ to 11.1$)$ & $11.0(9.3$ to 12.7$)$ & $11.7(10.0$ to 13.4$)$ & $\chi^{2}=3.49, P=0.06$ \\
\hline Parent reported symptoms† $(\mathrm{n}=3979)$ & $8.0(7.7$ to 8.3$)$ & $8.3(8.0$ to 8.6$)$ & $8.6(8.2$ to 8.9$)$ & $r=0.04, P=0.02$ \\
\hline Teacher reported symptoms† $(n=3081)$ & $6.1(5.7$ to 6.4$)$ & $6.3(5.9$ to 6.7$)$ & $6.6(6.2$ to 7.0$)$ & $r=0.03, \mathrm{P}=0.06$ \\
\hline Self reported symptoms† $(n=3799)$ & 10.2 (9.9 to 10.4$)$ & $10.4(10.1$ to 10.7$)$ & 10.6 (10.4 to 10.9$)$ & $r=0.04, \mathrm{P}=0.02$ \\
\hline
\end{tabular}

*Percentage ( $95 \%$ confidence interval); $\chi^{2}$ test.

tMean score $(95 \%$ confidence interval); Pearson correlation coefficient. 

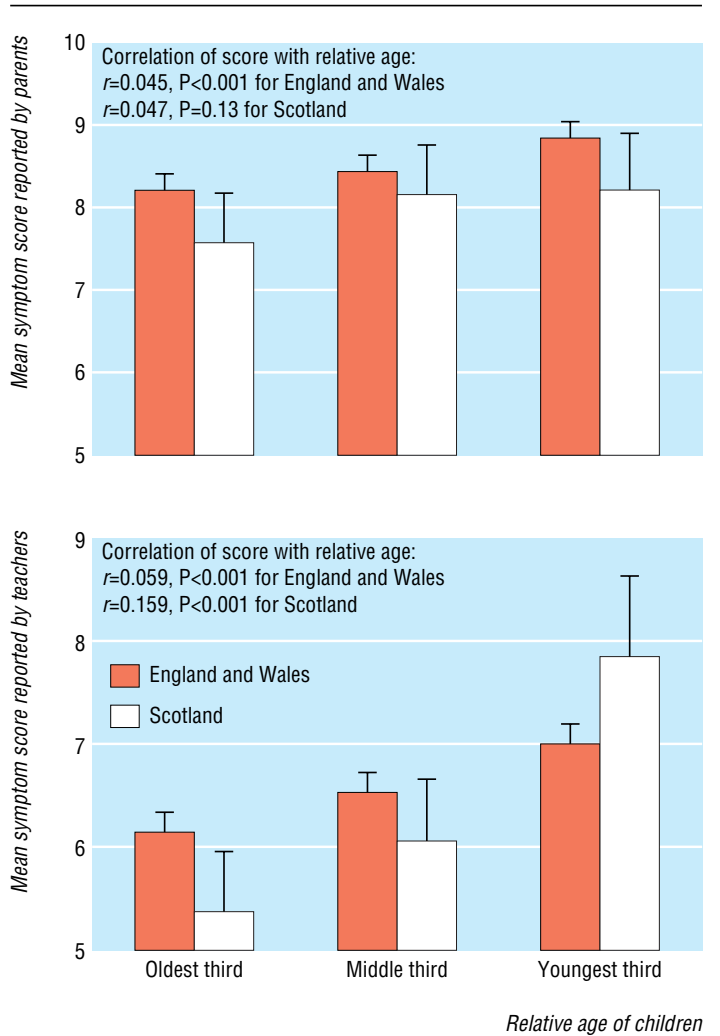

Association between relative age of children and mean symptom score on strengths and difficulties questionnaire reported by parents (top) and teachers (bottom). Bars are 95\% confidence intervals

shown)-for example, Scottish children born in January and February (and probably the youngest in their class) were at a disadvantage, whereas English and Welsh children born in the same months (but in the middle age band of their class) fared averagely.

\section{Discussion}

We found that relative age was an independent risk factor for psychopathology in 5-15 year olds, thereby confirming and extending previous findings. ${ }^{7}$ The link between relative age and psychopathology was robust in that it could be shown by using psychiatric diagnoses derived from research interviews as well as by using questionnaire scores derived from parents, teachers, and self report. The effect was not limited to the early school years and persisted after adjustment for other independent risk factors. Although the survey obtained a high response rate and the analyses were weighted for non-response by age, sex, and region, the sample will not have been totally representative. We find it hard, though, to imagine any non-response bias that would have mimicked our findings.

The national comparison between Scotland on the one hand and England and Wales on the other hand strongly indicated that the key explanatory variable was relative age rather than season of birth. It is common for an environmental "risk factor" to turn out to be either a marker for some previously unmeasured confounder or a consequence of the child's or parents' genotype. This is unlikely to be the case for relative age, which is unrelated to any other recognised risk factor and determined by an arbitrary cut-off imposed by the local or national government.

When thinking about individual children, the effects of relative age will generally be dwarfed by the much larger effects of well recognised risk factors such as family discord, adverse life events, or failure at school. ${ }^{8}$ Our findings do not provide clear guidance to schools and families who are trying to decide whether deferral of school entry will benefit an individual child. Despite being a modest effect at an individual level, the influence of relative age on psychopathology could nevertheless prove important at a public health level. An analogy may be helpful. A small change in the advertising or taxation of alcoholic drinks is not likely to be the sole cause of any one person developing severe alcohol related problems. However, even a small shift in the population's average alcohol consumption can have a disproportionate impact on the number at the top of the distribution. Similarly, steps to reduce the stresses associated with being the youngest in a class might result in only a small decrease in children's average level of psychopathology but could nevertheless result in a worthwhile reduction in the number of children with severe problems. If all children had the same risk of psychiatric disorder as that currently experienced by the oldest children in the class, the overall prevalence would fall from $9.0 \%$ (the average of oldest, middle, and youngest) to $8.3 \%$ (table), corresponding to a population attributable risk of $8 \%$. More than 8 million children aged 5-15 live in Britain, ${ }^{14}$ of whom approximately 750000 probably have a psychiatric disorder. ${ }^{8}$ Around 60000 of these cases of child psychiatric disorder might be prevented if the youngest and middle children in a school year were at no more risk than the oldest children.

The impact of relative age on psychopathology might be amenable to simple interventions. Several studies suggest that teachers often forget to make allowances for relative age, expecting too much of the younger children and being more likely to see them as failing. ${ }^{415}{ }^{16}$ Simple practical classroom interventions such as calling the register in birth order or grouping

\section{What is already known on this topic}

Being among the youngest children in a school year is associated with educational disadvantage

Teachers often forget to make allowances for differences in age within the school year

Younger children in a school year may be at greater psychiatric risk

\section{What this study adds}

It is "relative age" in the school year rather than "season of birth" that influences mental health

The tendency for the younger children in a school year to have more mental health problems is evident across different measures, raters, and age bands

Although the effect is weak at an individual level, it could prove important at a public health level 
children in the classroom by relative age may help to sensitise teachers to the age position of individual children within the class, thereby reducing the likelihood of unrealistic expectations being placed on younger children. Streaming children according to their relative age within each year group may also be helpful, as may allowing children who are struggling to repeat a year. In New Zealand, children spend between 12 months and 24 months in a reception or preparatory class, with progression to the next class being determined by the child's maturity and academic competence. Similarly, Scottish parents can choose to defer school entry for relatively young children who do not seem ready for school. The impact of such social experiments could be evaluated by randomised controlled trials.

Contributors: RG, JG, and TF all participated in the design, analysis, and writing up of this study. RG and TF were on the steering committee of the original survey. RG is guarantor of the study.

Funding: The original survey was funded by the British Department of Health.JG and TF are currently supported by Wellcome Trust research training fellowships. The guarantor accepts full responsibility for the conduct of the study, had access to the data, and controlled the decision to publish.

Competing interests: None declared.

Ethical approval: The ethical committee of the Institute of Psychiatry, King's College London approved the study.

1 Jinks PC. An investigation into the effect of date of birth on subsequent school performance. Educational Research 1964;6:220-5.
2 Russell R, Startup M. Month of birth and academic achievement. Journal of Personal Individual Experience 1986;7:839-46.

3 Wallingford EL, Prout HT. The relationship of season of birth and special education referral. Psychology in the School 2000;37:379-87.

4 Gledhill J, Ford T, Goodman R. Does season of birth matter? The relationship between age within the school year (season of birth) and educational difficulties amongst a representative general population sample of children and adolescents (aged 5-15) in Great Britain. Research in Education 2002;68:41-7.

5 Bergund G. A note on intelligence and season of birth. Br J Psychol 1967;58:147-51.

6 Sharp C. School entry and the impact of season of birth on attainment. Slough: National Foundation for Educational Research, 1995.

7 Mortimore P, Sammons P, Stoll L, Lewis D, Ecob R. School matters: the jun Mortimore P, Sammons P, Stoll L, Lew,
ior years. London: Open Books, 1988.

8 ior years. London: Open Books, 1988 . adolescents in Great Britain. London: Stationery Office, 2000.

9 Wright P, Takei N, Murray RM, Sham PC. Seasonality, prenatal influenza exposure, and schizophrenia. In: Susser E, Brown AS, Gorman JM, eds Prenatal exposures in schizophrenia. Washington, DC: American Psychiatric Press, 1999:89-112.

10 World Health Organization. The ICD-10 classification of mental and behavioural disorders: diagnostic criteria for research. Geneva: WHO, 1994.

11 Goodman R. Psychometric properties of the strengths and difficulties questionnaire (SDO). J Am Acad Child Adolesc Psychiatry 2001;40:1337-45.

12 Goodman R, Ford T, Richards H, Gatward R, Meltzer H. The development and well-being assessment: description and initial validation of an integrated assessment of child and adolescent psychopathology. J Child Psychol Psychiatry 2000;41:645-55.

13 Costello EJ, Burns BJ, Angold A, Leaf PJ. How can epidemiology improve mental health services for children and adolescents? J Am Acad Child Adolesc Psychiatry 1993;32:1106-14.

14 www.statistics.gov.uk/statbase/ssdataset.asp?vlnk $=5813 \&$ More $=\mathrm{Y}$ (accessed 6 Aug 2003).

15 Croll P, Moses D. (1985) One in five: the assessment and incidence of special educational needs. London: Routledge and Kegan Paul, 1985.

16 Tarnowski KJ, Anderson DF, Drabman RS, Kelly PA. Disproportionate referrals for child academic/behavior problems: replication and extension.J Consult Clin Psychol 1990;58:240-3.

(Accepted 9 July 2003) 